\title{
Quantificação de vitamina C e capacidade antioxidante de variedades cítricas
}

\author{
Quantification of vitamin $C$ and antioxidant capacity of citrus varieties
}

\author{
Meylene Aparecida Luzia COUTO ${ }^{1}$, Solange Guidolin CANNIATTI-BRAZACA*2
}

\section{Resumo}

A pesquisa teve por objetivo avaliar a variação do teor de vitamina $\mathrm{C}$ em diferentes variedades de laranjas e tangerinas de significado comercial no Brasil, como também medir a capacidade antioxidante destes citros. Foram realizadas análises para avaliação do índice de maturação no suco dos citros, como $\mathrm{pH}$, sólidos solúveis totais e acidez total titulável, e foram determinados os teores de vitamina C e capacidade antioxidante, sendo os dados comparados para verificar as diferenças pelo teste de Tukey $(\mathrm{p} \leq 0,05)$. As tangerinas apresentaram teores de vitamina $\mathrm{C}$ e capacidade antioxidante menores que as laranjas. Os teores de vitamina $\mathrm{C}$ variaram entre 21,47 e $84,03 \mathrm{mg}^{2}$ cido ascórbico.100 mL-1 de suco, apresentados, respectivamente, pela tangerina-murcote e laranja-natal. A tangerina-murcote também apresentou a menor capacidade antioxidante (12,78\%) e a maior capacidade antioxidante foi da laranja-lima (66,24\%). Conclui-se que as diferentes variedades de laranjas e tangerinas possuem variação significativa nas variáveis analisadas, sendo que as laranjas apresentaram os maiores teores de vitamina C e capacidade antioxidante.

Palavras-chave: citros; vitamina C; polifenóis; capacidade antioxidante.

\begin{abstract}
The objective of this research was to evaluate the variation of vitamin $\mathrm{C}$ in different commercial varieties of oranges and tangerines in Brazil and to measure the antioxidant capacity of these citrus. The analyses done for the evaluation of the maturity index of the citrus juices were $\mathrm{pH}$, total soluble solids, and total tritimetric acidity. The antioxidant capacity of vitamin $\mathrm{C}$ was also quantified. The data obtained was submitted to statistical analysis using the Tukey test $(\mathrm{p} \leq 0.05)$. The tangerines presented lower levels of vitamin $\mathrm{C}$ and lower antioxidant capacity than

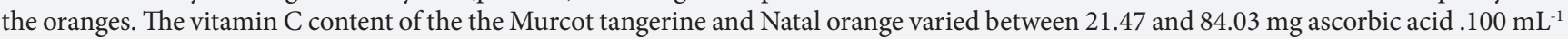
juice, respectively. The Murcot tangerine exhibited the smallest antioxidant capacity (12.78\%), and the sweet orange exhibited the largest antioxidant capacity (66.24\%). The conclusion was that the different varieties of oranges and tangerines had a significant variation in the variables analyzed, and that the oranges presented the largest levels of vitamin $\mathrm{C}$ and the largest antioxidant capacity.

Keywords: citrus; vitamin C; polyphenols; food antioxidants.
\end{abstract}

\section{Introdução}

A citricultura é o ramo que mais se destaca na fruticultura mundial, o que faz dos citros as frutas mais produzidas no mundo. $\mathrm{O}$ cultivo de citros foi introduzido no Brasil ainda no período colonial, e desde então passou a ter grande importância nos hábitos de consumo de nossa população. Porém, o País iniciou seu avanço rumo à liderança mundial na produção de laranja somente a partir de 1960, e desde meados dos anos 80 assume a primeira posição na produção dessa fruta (USP, 2004).

O Brasil obteve produção na safra de 2005/2006 de 18,238 milhões de toneladas métricas, equivalente a 38,72\% da safra mundial de laranja. A laranja representa $49 \%$ da produção brasileira de frutas. Entre a citricultura regional, o principal polo produtor de laranja é o Estado de São Paulo, responsável por 80,4\% da produção nacional em 2006 (IFNP, 2007; NEVES; LOPES, 2005).
Os citros, principalmente laranjas e tangerinas, fazem parte da dieta dos brasileiros. Além de serem importante fonte de vitaminas e fibras, as frutas e sucos cítricos recentemente vêm sendo reconhecidos por conterem metabólitos secundários incluindo antioxidantes como ácido ascórbico, compostos fenólicos, flavonoides, limonoides que são importantes para a nutrição humana (JAYAPRAKASHA; PATIL, 2007).

Os antioxidantes são compostos químicos que podem prevenir ou diminuir os danos oxidativos de lipídios, proteínas e ácidos nucleicos causados por espécies de oxigênio reativo, que incluem os radicais livres, ou seja, os antioxidantes possuem a capacidade de reagir com os radicais livres e assim restringir os efeitos maléficos ao organismo. Os citros, assim como muitas frutas, são ricos em substâncias antioxidantes que ajudam a diminuir a incidência de doenças degenerativas, como o câncer, as doenças cardiovasculares, inflamações, disfunções cerebrais,

Recebido para publicação em 26/9/2007

Aceito para publicação em 4/3/2008 (002885)

${ }^{1}$ Ciências dos Alimentos, Escola Superior de Agricultura "Luiz de Queiroz", Av. Pádua Dias, 11, CP 9, CEP 13418-900, Piracicaba - SP, E-mail: malcouto@esalq.usp.br

2 Departamento de Agroindústria, Alimentos e Nutrição, Escola Superior de Agricultura "Luiz de Queiroz" - ESALQ, Universidade de São Paulo - USP, Av. Pádua Dias, 11, CP 9, CEP 13418-900, Piracicaba - SP, E-mail: sgcbraza@esalq.usp.br

${ }^{*}$ A quem a correspondência deve ser enviada 
e a retardar o envelhecimento precoce (PIMENTEL; FRANCKI; GOLLÜCKE, 2005).

As frutas e vegetais contêm muitos compostos com potencial atividade antioxidante, como vitaminas $\mathrm{C}$ e $\mathrm{E}$, carotenoides, clorofilas, e uma variedade de antioxidantes fitoquímicos como compostos fenólicos simples, glicosídeos e flavonoides (PELLEGRINI et al., 2007).

A maioria dos antioxidantes presentes em citros é vitamina $\mathrm{C}$ e polifenóis, principalmente flavonoides. A vitamina $\mathrm{C}$ proporciona proteção contra a oxidação descontrolada no meio aquoso da célula, devido ao seu alto poder redutor. Os polifenóis são substâncias com grande poder de neutralizar as moléculas de radicais livres (KLIMCKAC et al., 2007; JAYAPRAKASHA; PATIL, 2007).

Vista a importância que os citros representam à economia e à dieta dos brasileiros, e a importância de pesquisas por antioxidantes naturais, este trabalho teve o objetivo de avaliar a variação do teor de vitamina $\mathrm{C}$ e capacidade antioxidante nas diferentes variedades de laranjas e tangerinas de significado comercial no Brasil.

\section{Material e métodos}

\subsection{Material}

Foram utilizadas diferentes variedades de citros de significado comercial, sendo estes de tamanho médio, pesando em média 154 g laranja-pera, 150 g laranja-lima, $180 \mathrm{~g}$, laranja-baía, 160 g laranja-natal, 162 g laranja-valência, $171 \mathrm{~g}$ tangerina-murcote e 184 g tangerina-poncã. As variedades utilizadas foram provenientes dos seguintes locais:

- Laranja-pera: Fazenda São Pasquale (Iperó - SP);

- Laranja-lima: Fazenda Ana Maria (Sorocaba - SP);

- Laranja-baía: Fazenda Ana Maria (Sorocaba - SP);

- Laranja-natal: F.C. Frutas Conchal Sociedade Ltda. (Mogi Guaçu - SP);

- Laranja-valência: F.C. Frutas Conchal Sociedade Ltda. (Mococa - SP);

- Tangerina-murcote: Fazenda Bela Vista (São Pedro do Turvo - SP); e

- Tangerina-poncã: Fazenda São Thomaz (Porto Feliz - SP).

\subsection{Metodologia}

\section{Caracterização das amostras}

O experimento foi feito entre outubro de 2006 e maio de 2007, devido às épocas específicas de disponibilidade das variedades de citros, os quais foram obtidos diretamente com os produtores logo após a colheita.

A amostragem foi realizada ao acaso, foram utilizadas três amostras de tamanho médio de cada variedade, o suco de cada amostra foi extraído manualmente e depois homogeneizado para realização das análises.

As análises físico-químicas para avaliação do índice de maturação foram realizadas no suco, após a sua homogeneização, e constaram das seguintes variáveis: $\mathrm{pH}$ determinado em Phâmetro (MS Tecnopon, modelo mPA 210, Piracicaba, Brasil); sólidos solúveis totais $\left({ }^{\circ} \mathrm{Brix}\right)$ em refratômetro (Abbe Tipo WYA); acidez total titulável (\% de ácido cítrico.100 $\mathrm{mL}^{-1} \mathrm{de}$ suco), determinada por titulometria com solução de hidróxido de sódio $0,1 \mathrm{~N}$ e fenolftaleína como indicador (INSTITUTO ADOLFO LUTZ, 1985).

\section{Determinação de vitamina $C$}

A extração da vitamina $C$ foi realizada a partir de $5 \mathrm{~mL}$ de suco, ao qual foram adicionados $50 \mathrm{~mL}$ de ácido oxálico 0,5 g. $100 \mathrm{~mL}^{-1}$. A titulação da quantidade de vitamina $\mathrm{C}$ (ácido ascórbico) nas amostras foi feita utilizando o indicador 2,6-diclobenzenoindofenol (JACOBS, 1958; LEME Jr.; MALAVOLTA, 1950).

\section{Capacidade antioxidante}

A determinação da capacidade antioxidante dos citros foi feita em solução de etanol pelo método Brand-Williams (BRAND-WILLIAMS; CUVELIER; BERSET, 1995) adaptado, com 2,2'difenil-1-picril-hidrazila (DPPH). As amostras foram preparadas e medidas separadamente em triplicatas.

O suco dos citros foi diluído em etanol, em proporção de $0,8 \mathrm{~g}$ de suco/25 mL etanol. Em tubo de ensaio, colocouse $500 \mu \mathrm{L}$ do suco da amostra diluído, $300 \mu \mathrm{L}$ de solução de $\mathrm{DPPH}(0,5 \mathrm{mM})$ e $3 \mathrm{~mL}$ de etanol. O controle foi preparado conforme procedimento acima, sem adição de extrato, e etanol foi utilizado para correção da linha de base. As soluções foram deixadas em repouso por 45 minutos em ambiente escuro, com posterior leitura em espectrofotômetro (Du Series 600, Bechman Instruments, Fullerton, Estados Unidos) a $517 \mathrm{~nm}$.

O percentual de decréscimo na absorbância foi medido e a capacidade de sequestrar radicais livres foi calculada com base no decréscimo da absorbância observada. A capacidade antioxidante foi expressa como a porcentagem DPPH reduzido, expressa pela Equação 1:

(Abs do controle - Abs da amostra)*100/Abs do controle

\section{Delineamento estatístico}

O experimento foi realizado ao acaso, em triplicata. As análises foram realizadas em triplicata e os dados analisados por teste F e, se significativo, por teste de Tukey $(\mathrm{p} \leq 0,05)$. Para verificar a correlação entre a capacidade antioxidante e o teor de vitamina C, foi realizada a correlação de Pearson. O programa estatístico utilizado foi o STATISTICAL (SAS, 1996).

\section{Resultados e discussão}

São apresentados os dados de $\mathrm{pH}$, acidez titulável, sólidos solúveis e ratio das amostras avaliadas na Tabela 1. 
A Tabela 1 mostra os resultados referentes ao estado de maturação das frutas cítricas analisadas. Os valores de $\mathrm{pH}$ dos sucos de laranjas e tangerinas variaram entre 3,20 e 5,43 . Os sólidos solúveis tiveram variação entre 9,11 e $14,33^{\circ}$ Brix. A acidez titulável, expressa em porcentagem de ácido cítrico por $100 \mathrm{~mL}$ de suco, variou entre 0,23 e 1,48, sendo a laranja-lima a que apresentou o menor teor de acidez, assim como verificado por Ramalho (2005). O consumo da maioria das laranjas e tangerinas depende da diminuição do teor de acidez até um ponto em que o seu suco se torne agradável ao paladar, sendo que o ácido cítrico é o seu principal ácido orgânico.

O ratio é a relação entre o teor de sólidos solúveis ( ${ }^{\circ}$ Brix) e o teor de ácidos tituláveis. Ele é o indicador utilizado para determinar o estágio de maturação, determinando o balanço do sabor doce:ácido. Segundo Viégas (1991), a faixa de ratio pode variar entre 6 e 20 , sendo o intervalo de 15 a 18 o preferido pelos consumidores. A indústria inicia o processamento com valor entre 12 e 13.

No Brasil, verifica-se a preferência por sucos com ratio acima de 14. A evolução do ratio em laranjas doces no Estado de São Paulo, em parte pode ser explicada pela relação portaenxerto/copa, idade das árvores, florada e produtividade, além da variação do clima de ano para ano (VOLPE; SHOFFELE; BARBOSA, 2002). Já Sartori et al. (2002), considera como maduros e adequados para o consumo, frutos que apresentam SST/ATT entre 8,8 e 15,4 .

No presente trabalho, as frutas cítricas estudadas apresentaram variação significativa nos valores de ratio, estando entre 6,65 e 38,82 . Analisando os resultados, verificou-se que a laranja-lima apresentou maior ratio e bem acima do valor mostrado, devido à baixa acidez, apresentada pelos autores para adequação ao consumo, podendo então ter tido uma colheita tardia estando fora dos padrões normalmente utilizados. Isto pode ser mostrado por Ramalho (2005), em estudo que correlaciona positivamente o parâmetro ratio com atributos de sabor avaliados por análises sensoriais, como maduro $(0,71) \mathrm{e}$ passado $(0,93)$, o que mostra que, conforme o ratio aumenta, esses atributos tornam-se mais perceptíveis para o provador.

Conforme Calore e Vieites (2003), há diferenças nas proporções açúcar/ácido entre variedades do mesmo produto e mesmo dentro da mesma variedade cultivada em diferentes condições, conforme foi encontrado para as amostras analisadas.

A relação entre o teor de sólidos solúveis totais e acidez total titulável expressa o balanço entre esses componentes no suco, conferindo-lhe o sabor. Esse sabor pode ser mais ou menos ácido em função da região produtora ou das características do cultivar (TAVARES, 2003), o que caracteriza cada citro utilizado no estudo.

Os teores de ácido ascórbico quantificados estão demonstrados na Tabela 2. As tangerinas apresentaram menor teor de ácido ascórbico que as laranjas.

Entre as tangerinas, a variedade Murcote apresentou menor teor que a Poncã. A tangerina-poncã apresentou menor teor de vitamina $\mathrm{C}$ que o descrito para ela na Tabela Brasileira de Composição de Alimentos (UNICAMP, 2006), que foi de $41,8 \mathrm{mg}$ de ácido ascórbico.100 $\mathrm{mL}^{-1}$ de suco. A tangerinamurcote apresentou teor de vitamina $\mathrm{C}$ bem próximo do obtido por Moreira (2004), em estudo no qual o suco continha 25,94 mg AA. $100 \mathrm{~mL}^{-1}$ no tempo zero de armazenamento.

As diferentes variedades de laranjas apresentaram variação significativa na quantidade de vitamina $\mathrm{C}$, sendo que a laranjapera apresentou o menor valor (62,50 mg AA. $100 \mathrm{~mL}^{-1}$ de suco) e a laranja-natal o maior (84,03 mg AA. $100 \mathrm{~mL}^{-1}$ de suco).

Conforme a tabela TACO (UNICAMP, 2006), o teor de ácido ascórbico na laranja-baía e na laranja-pera são 94,50 e $73,30 \mathrm{mg} .100 \mathrm{~mL}^{-1}$ de suco, respectivamente; sendo estes valores maiores que os apresentados nas laranjas analisadas. Em relação à laranja-lima, o teor de vitamina $\mathrm{C}$ foi maior nas amostras analisadas em comparação com os resultados descritos na tabela (UNICAMP, 2006), que foi 41,3 mg AA.100 $\mathrm{mL}^{-1} \mathrm{de}$ suco. Essas variações podem ocorrer entre as cultivares, local e ano de produção, além do grau de maturação dos frutos.

Pellegrini et al. (2007), avaliando o teor de ácido ascórbico de algumas frutas e vegetais, obtiveram teor de 57,5 mg AA.100 g $\mathrm{g}^{-1}$ para laranja, valor esse menor que o encontrado (Tabela 2) para laranja, quando foi considerado o teor de vitamina $\mathrm{C}$ delas.

Lim, Y. Y., Lim, T. T. e Tee (2007), em estudo comparativo de frutas tropicais, encontraram valor próximo ao obtido

Tabela 1. Valores de $\mathrm{pH}$, acidez titulável, sólidos solúveis e ratio das variedades de citros.

\begin{tabular}{lcccc}
\hline \multicolumn{1}{c}{ Amostra } & $\mathrm{pH}$ & Acidez titulável (\%) & Sólidos solúveis ( ${ }^{\circ}$ Brix $)$ & Ratio \\
\hline Tangerina ponkã & $4,05 \pm 0,00^{1 \mathrm{~b} 2}$ & $0,80 \pm 0,00^{\mathrm{c}}$ & $12,87 \pm 0,06^{\mathrm{b}}$ & $15,99^{\mathrm{bc}}$ \\
Tangerina murcote & $3,85 \pm 0,04^{\mathrm{c}}$ & $0,80 \pm 0,00^{\mathrm{c}}$ & $14,33 \pm 0,12^{\mathrm{a}}$ & $17,81^{\mathrm{b}}$ \\
Laranja pêra & $3,66 \pm 0,02^{\mathrm{d}}$ & $1,14 \pm 0,12^{\mathrm{b}}$ & $10,60 \pm 0,09^{\mathrm{c}}$ & $9,37^{\mathrm{bc}}$ \\
Laranja lima & $5,43 \pm 0,06^{\mathrm{a}}$ & $0,23 \pm 0,06^{\mathrm{d}}$ & $9,11 \pm 0,54^{\mathrm{e}}$ & $38,82^{\mathrm{a}}$ \\
Laranja natal & $3,53 \pm 0,01^{\mathrm{e}}$ & $1,24 \pm 0,06^{\mathrm{b}}$ & $10,89 \pm 0,14^{\mathrm{c}}$ & $8,79^{\mathrm{bc}}$ \\
Laranja valência & $3,62 \pm 0,01^{\mathrm{d}}$ & $0,80 \pm 0,00^{\mathrm{c}}$ & $10,73 \pm 0,14^{\mathrm{c}}$ & $13,33^{\mathrm{bc}}$ \\
Laranja bahia & $3,20 \pm 0,01^{\mathrm{f}}$ & $1,48 \pm 0,12^{\mathrm{a}}$ & $9,81 \pm 0,14^{\mathrm{d}}$ & $6,67^{\mathrm{c}}$ \\
\hline
\end{tabular}

${ }^{1}$ Média \pm desvio padrão de três repetições; $\mathrm{e}^{2}$ Médias com letras minúsculas diferentes nas colunas diferem significativamente ( $\mathrm{p} \leq 0,05$ ). 
neste trabalho (Tabela 2) no teor de ácido ascórbico da laranja (67 mg.100 g $\mathrm{g}^{-1}$.

Em estudo sobre compostos bioativos de diferentes variedades de frutas cítricas cultivadas em Taiwan, Wang, Chuang e $\mathrm{Ku}$ (2007) encontraram valores superiores no teor de ácido ascórbico das frutas em relação às frutas deste estudo. Isso porque o teor de vitamina $\mathrm{C}$ nos alimentos é variável de acordo com a região de cultivo, clima, época de colheita, mesmo sendo a mesma variedade.

As análises relacionadas à capacidade antioxidante dos citros mostraram haver variação significativa da capacidade antioxidante entre as diferentes variedades de laranjas e tangerinas de significado comercial. O suco da tangerinaMurcote apresentou menor capacidade antioxidante (12,78\%) e de laranja-lima maior $(66,24 \%)$. Os dados mostram que as tangerinas apresentam menor capacidade antioxidante que as laranjas.

Conforme Pimentel, Francki e Gollücke (2005), há uma vasta evidência epidemiológica para os efeitos benéficos que o consumo de sucos cítricos exerce na saúde humana. Estes efeitos são atribuídos às substâncias que reduzem radicais livres e que possuem atividades antioxidantes, principalmente ácido ascórbico, flavonoides, carotenoides e derivados fenólicos. Segundo Jayaprakasha e Patil (2007), variações na capacidade antioxidante de diferentes extratos podem ser atribuídas à diferença na composição química como fenólicos, ácido ascórbico e carotenoides, o que pode ter ocorrido entre as diferentes cultivares de laranjas e tangerinas (Tabela 3).

Essa capacidade antioxidante (Tabela 3) provavelmente se deve aos compostos fenólicos e ao ácido ascórbico que são os principais antioxidantes dos citros. Os compostos fenólicos são capazes de reduzir radicais livres e quelar metais, enquanto o ácido ascórbico pode ter um papel pro-oxidante na presença de metais de transição (HALLIWELL, 2001).

Nas análises referentes à capacidade antioxidante feita pelo método $\mathrm{DPPH}$, o declínio da coloração da solução indica a redução do DPPH pela substância antioxidante do extrato ou amostra utilizada, devido à alta habilidade de doação de hidrogênio pelos compostos antioxidantes (JAYAPRAKASHA; PATIL, 2007).

A atividade antioxidante de diferentes amostras é difícil de ser comparada, pois os autores utilizam diferentes diluições das amostras para a realização da análise, já que cada amostra apresenta um poder antioxidante diferente. Além disso, as análises e formas para expressar os resultados são diversas. Stratil, Klejdus e Kubáñ (2007) relatam que a comparação de resultados referentes à capacidade antioxidante publicados nos métodos individuais e entre publicações do mesmo método é frequentemente problemática.

Os resultados encontrados por Pellegrini et al. (2007) indicam que a laranja é uma excelente fonte de fenólicos e ácido ascórbico, além de possuir potencial antioxidante extremamente alto, sendo a contribuição do ácido ascórbico e compostos fenólicos de 76\% no valor da atividade antioxidante total da laranja.

Lim, Y. Y., Lim, T. T. e Tee (2007) estudaram a capacidade antioxidante de várias frutas tropicais e verificaram que mamãopapaia, carambola e goiaba possuem poder antioxidante maior que a laranja, sendo que esta possui $\mathrm{IC}_{50}$ de $5,4 \mathrm{mg} \cdot \mathrm{ml}^{-1}$, provavelmente devido à composição diferente. Gardner et al. (2000) observaram que tanto vitamina C quanto polifenóis totais em sucos de frutas estão fortemente relacionados com a capacidade antioxidante. Jayaprakasha e Patil (2007) relatam que alguns dos extratos de frutas cítricas estudadas podem exibir propriedades antioxidantes comparáveis com antioxidantes comerciais sintéticos. Esses autores reforçam a veracidade dos resultados encontrados, pois com a alteração da composição das laranjas e tangerinas ocorre a alteração da capacidade antioxidante.

Conforme a Tabela 4, não existe correlação entre vitamina C e capacidade antioxidante, mostrando a relação proporcional entre estas variáveis. No entanto, conforme os resultados obtidos, essa relação não foi significativa. Este resultado pode ter ocorrido devido ao efeito de outros compostos que são importantes para a capacidade antioxidante, além da vitamina C (YU et al., 2002).

Tabela 2. Teores de ácido ascórbico das variedades de citros.

\begin{tabular}{lc}
\hline \multicolumn{1}{c}{ Amostra } & Ácido ascórbico $\left(\mathrm{mg} \cdot 100 \mathrm{~mL}^{-1}\right.$ suco $)$ \\
\hline Tangerina ponkã & $32,47 \pm 1,79^{1 \mathrm{~d} 2}$ \\
Tangerina murcote & $21,47 \pm 1,11^{\mathrm{e}}$ \\
Laranja pêra & $62,50 \pm 0,96^{\mathrm{c}}$ \\
Laranja lima & $64,58 \pm 0,46^{\mathrm{c}}$ \\
Laranja natal & $84,03 \pm 3,18^{\mathrm{a}}$ \\
Laranja valência & $78,47 \pm 1,20^{\mathrm{b}}$ \\
Laranja bahia & $80,03 \pm 1,03^{\mathrm{ab}}$ \\
\hline
\end{tabular}

${ }^{1}$ Média \pm desvio padrão de três repetições; $\mathrm{e}^{2}$ Médias com letras minúsculas diferentes nas colunas diferem significativamente $(\mathrm{p} \leq 0,05)$.

Tabela 3. Capacidade antioxidante das variedades de citros.

\begin{tabular}{lc}
\hline \multicolumn{1}{c}{ Amostra } & Capacidade antioxidante $(\%)$ \\
\hline Tangerina ponkã & $29,30 \pm 1,43^{1 \mathrm{~d} 2}$ \\
Tangerina murcote & $12,78 \pm 3,47^{\mathrm{e}}$ \\
Laranja pêra & $49,15 \pm 3,32^{\mathrm{b}}$ \\
Laranja lima & $66,24 \pm 2,35^{\mathrm{a}}$ \\
Laranja natal & $51,28 \pm 2,03^{\mathrm{b}}$ \\
Laranja valência & $41,84 \pm 0,49^{\mathrm{c}}$ \\
Laranja bahia & $60,32 \pm 2,62^{\mathrm{a}}$ \\
\hline
\end{tabular}

${ }^{1}$ Média \pm desvio padrão de três repetições; $\mathrm{e}^{2}$ Médias com letras minúsculas diferentes nas colunas diferem significativamente $(\mathrm{p} \leq 0,05)$.

Tabela 4. Valores de correlação para as variáveis analisadas.

\begin{tabular}{lcc}
\hline & & Correlação de Pearson \\
\hline $\mathrm{pH}$ & Vitamina C & $0,225585^{\text {ns }}$ \\
Vitamina C & Capacidade antioxidante & $0,807893^{\text {ns }}$ \\
\hline
\end{tabular}




\section{Conclusões}

De acordo com os resultados obtidos nestas condições de avaliação, com a colheita entre outubro de 2006 e maio de 2007, conclui-se que as diferentes variedades de laranjas e tangerinas possuem variação significativa nos teores de vitamina $\mathrm{C}$ e capacidade antioxidante, e que as laranjas são mais ricas em vitamina $\mathrm{C}$ e possuem maior poder antioxidante que as tangerinas.

\section{Agradecimentos}

A Raphael Juliano Ltda e F.C.Frutas Conchal Sociedade Ltda pela doação das laranjas e tangerinas utilizadas nesta pesquisa.

\section{Referências bibliográficas}

BRAND-WILLIAMS, W.; CUVELIER, M. E.; BERSET, C. Use of a free radical method to evaluate antioxidant activity. LebensmittelWissenschaft \& Technologie, v. 28, n. 1, p. 25-30, 1995.

CALORE, L.; VIEITES, R. L. Conservação de pêssegos "Biuti" por irradiação. Ciência e Tecnologia de Alimentos, v. 23, supl., p. 53-57, 2003.

GARDNER, P. T. et al. The relative contributions of vitamin C, carotenoids and phenolics to the antioxidant potential of fruit juices. Food Chemistry, v. 68, n. 4, p. 471-474, 2000.

HALLIWELL, B. Vitamin C and genomic stability. Mutation Research: Fundamental and Molecular Mechanisms of Mutagenesis, v. 475, n. 1-2, p. 29-35, 2001.

INSTITUTO ADOLFO LUTZ - IAL. Normas analíticas, métodos químicos e físicos para análise de alimentos. 3 ed. São Paulo, 1985. 533 p. (v. 1)

INSTITUTO FNP - IFNP. Agrianual 2007: anuário da agricultura brasileira. São Paulo, 2006. 516 p.

JACOBS, M. B. The chemical analysis of foods and food products. New York: Van Nostrand, 1958. 979 p.

JAYAPRAKASHA, G. K.; PATIL, B. S. In vitro evaluation of the antioxidant activities in fruit extracts from citron and blood orange. Food Chemistry, v. 101, n. 1, p. 410-418, 2007.

KLIMCZAK, I. et al. Effect of storage on the content of polyphenols, vitamin $\mathrm{C}$ and the antioxidant activity of orange juices. Journal of Food Composition and Analysis, v. 20, n. 3-4, p. 313-322, 2007.

LEME Jr., J.; MALAVOLTA, E. Determinação fotométrica de ácido ascórbico. Anais da Escola Superior de Agricultura Luiz de Queiroz, v. 7, p. 115-129, 1950.

LIM, Y. Y.; LIM, T. T.; TEE, J. J. Antioxidant properties of several tropical fruits: a comparative study. Food Chemistry, v. 103, n. 3 , p. 1003-1008, 2007.

MOREIRA, R. C. Processamento mínimo de tangor "Murcott": caracterização fisiológica e recobrimentos comestíveis. Piracicaba,
2004. 72 p. Dissertação (Mestrado em Fitotecnia) - Universidade de São Paulo - USP.

NEVES, M. F.; LOPES, F. F. Estratégias para a laranja no Brasil. São Paulo: Ed Atlas, 2005. 225 p.

PELLEGRINI, N. et al. Evaluation of antioxidant capacity of some fruit and vegetable foods: efficiency of extraction of a sequence of solvents. Journal of the Science of Food and Agriculture, v. 87, n. 1, p. 103-111, 2007.

PIMENTEL, C. V. M. B.; FRANCKI, V. M.; GOLLÜCKE, A. P. B. Alimentos funcionais: introdução as principais substâncias bioativas em alimentos. São Paulo: Ed. Varela, 2005. 95 p.

RAMALHO, A. S. T. M. Sistema funcional de controle de qualidade a ser utilizado como padrão na cadeia de comercialização de laranja Pêra (Citrus sinensis L. Osbeck). Piracicaba, 2005. 91 p. Dissertação (Mestrado em Ciência e Tecnologia de Alimentos) Universidade de São Paulo - USP.

SARTORI, I. A. et al. Maturação de frutos de seis cultivares de laranjasdoces na depressão central do Rio Grande do Sul. Revista Brasileira de Fruticultura, v. 24, n. 2, p. 364-369, 2002.

STRATIL, P.; KLEJDUS, B.; KUBÁÑ, V. Determination of phenolic compounds and their antioxidant activity in fruits and cereals. Talanta, v. 71, n. 4, p. 1741-1751, 2007.

STATISTICAL ANALYSIS SYSTEM INSTITUTE - SAS. SAS/QC software: usage and reference. Version 6. 2 ed. Cary, 1996. (CDROM).

TAVARES, S. Maturação e conservação do Tangor "Murcote" (Citrus reticulata blanco x C. sinensis Osbeck) e de Lima ácida "Tahiti” (Citrus latifolia Tanaka) sob efeito de biorreguladores. Piracicaba, 2003. 115 p. Tese (Doutorado em Fitotecnia) - Universidade de São Paulo - USP.

UNIVERSIDADE DE SÃO PAULO - USP. Citros colorem Sudeste brasileiro de verde e laranja. Visão Agrícola, v. 1, n. 2, p. 90-99, 2004.

UNIVERSIDADE ESTADUAL DE CAMPINAS - UNICAMP. Tabela Brasileira de Composição de Alimentos: TACO. 2 ed. Campinas, 2006. Disponível em: <http://www.unicamp.br/nepa/taco/contar/ taco_versao2.pdf>. Acesso em: 20 maio 2007.

VIÉGAS, F. C. P. A citricultura brasileira. 2 ed. Campinas: Cargil, 1991.

VOLPE, A. C.; SHOFFELE, R.; BARBOSA, J. C. Influência da soma térmica e da chuva durante o desenvolvimento de laranjas "Valência" e "Natal" na relação entre sólidos solúveis e acidez e no índice tecnológico do suco. Revista Brasileira de Fruticultura, v. 24, n. 2, p. 436-441, 2002.

YU, L. et al. Free radical scavenging properties of wheat extracts. Journal of Agricultural and Food Chemistry, v. 50, n. 6, p. 1619-1624, 2002.

WANG, Y.; chuang, y. c.; ku, Y. H. Quantitation of bioactive compounds in citrus fruits cultivated in Taiwan. Food Chemistry, v. 102, n. 4, p. 1163-1171, 2007. 